\title{
Numerical simulation of motor neuron excitability in a transgenic mouse model of familial amyotrophic lateral sclerosis
}

\author{
N. Marzocchi ${ }^{1,2}$, S. Severi ${ }^{1}$, M. Pieri ${ }^{3,4}$, C. Zona ${ }^{3,4}$ \& S. Cavalcanti ${ }^{1}$ \\ ${ }^{1}$ Cellular and Molecular Engineering Laboratory, D.E.I.S., \\ University of Bologna, Cesena, Italy \\ ${ }^{2}$ Department of Human and General Physiology, \\ University of Bologna, Italy \\ ${ }^{3}$ Department of Neuroscience, University of Rome "Tor Vergata", Italy \\ ${ }^{4}$ Fondazione S. Lucia, I.R.C.C.S., Rome, Italy
}

\begin{abstract}
Amyotrophic lateral sclerosis (ALS) is a neurodegenerative disease characterized by the selective degeneration of motor neurons. Mutations of the $\mathrm{Cu}, \mathrm{Zn}$ superoxide dismutase (SOD1) account for $20 \%$ of the familial cases of ALS. Transgenic mice overexpressing the mutated human SOD1 enzyme develop phenotypic and pathological symptoms resembling ALS in humans. Previous studies showed that, in this transgenic model of ALS, expression of the human G93A-mutant SOD1 gene does not affect passive membrane properties, whereas it induces a different excitability with respect to control and SOD1 motor neurons. To investigate which modifications at the ionic current level can produce the observed phenomenon, we developed a numerical simulator of the electrical activity of mouse spinal motor neuron. The simulator was based on a mathematical model including a Hodgkin-Huxley (HH)-type parallel conductance membrane model and a lumped fluid compartment model accounting for the activity of a cytosolic calcium-binding buffer. The equations describing voltage-dependent fast sodium current $\left(\mathrm{I}_{\mathrm{Na}}\right)$ were modified from a conventional Hodgkin-Huxley formulation, and the parameters were adjusted in order to fit experimental data from non-transgenic mouse motor neurons. We then introduced minimal changes to other model parameters in order to match available SOD1 current-clamp electrophysiological data. The model is able to reproduce AP waveform properties as well as the relation between firing frequency and injected current measured in SOD1 motor neurons. This model offers a powerful investigative tool, making it possible to identify the current modifications which could produce the observed effects on G93A motor neurons excitability.
\end{abstract}

Keywords: biological system modeling, motor neuron, firing properties, Hodgkin-Huxley models, ALS. 


\section{Introduction}

Amyotrophic lateral sclerosis (ALS) is a progressive neurodegenerative disease characterized by the selective loss of cortical and spinal motor neurons, leading to skeletal muscle atrophy, paralysis and death. Mutations in the gene coding for human $\mathrm{Cu}, \mathrm{Zn}$ superoxide-dismutase (SOD1) have been reported for about $20 \%$ of the familial form of ALS [1].

Transgenic mice overexpressing the mutated (Gly93 $\rightarrow$ Ala) human SOD1 enzyme develop phenotypic and pathological symptoms resembling ALS in humans whereas transgenic mice expressing wild-type human SOD1 do not [2]. Previous studies on spinal motor neurons excitability in this transgenic model of ALS showed that expression of the human G93A-mutant SOD1 gene induces increased firing frequency and shorter action potential (AP) duration with respect to control and SOD1 motor neurons [3]. This is in agreement with transcranial magnetic stimulation (TMS) studies showing that, in ALS patients, increased excitability of corticomotoneurons contribute to motor cortex hyperexcitability [4]. Such results suggest that an altered excitability of motor neurons can contribute to the pathogenesis of motor neuron degeneration, which ultimately leads to cell death. In order to deepen the knowledge about ALS disease it is therefore crucial to identify which mechanisms underlie the observed motor neurons hyperexcitability.

It is well known that several distinct ionic currents determine membrane excitability and AP properties. Previous studies analyzed the correlation between AP waveform and ionic current changes in motor neurons [5,6]. However, little is known about ionic current alterations underlying the observed motor neuron hyperexcitability in ALS. Due to complex interactions between the various ionic currents, it is difficult to study in vivo these problems. Numerical simulation of neuronal excitability allows one to easily evaluate the effects of ionic current alterations on firing properties, and therefore it can help to characterize possible involved mechanisms that can be confirmed by more targeted experimental studies.

As a first step in our analysis of ALS neuron hyperexcitability, in this work we present a computer model for the electrical activity of mouse spinal motor neuron, providing a useful tool for analyzing the role of ionic currents in these neurons.

\section{Methods}

\subsection{Firing properties of SOD1 spinal motor neurons}

Current clamp electrophysiological experiments have been performed on spinal motor neurons of transgenic mice expressing the human wild-type protein (SOD1). The detailed description of experimental procedures and the results are reported in Pieri et al. (2003) [3]. The characteristics of action potential morphology (threshold, amplitude, duration, rate of rise and rate of repolarization) were derived from the first spike of the AP discharges elicited on 
the injection of a $+200 \mathrm{pA}$ depolarizing current. The firing frequency was calculated as the inverse of the first interspike interval evoked by four levels of depolarizing current $(+200,+300,+400$, and $+500 \mathrm{pA})$.

\subsection{Sodium current model}

Sodium current was modeled using the equation:

$$
I_{N a}=\bar{g}_{N a} m^{3} h\left(V-E_{N a}\right)
$$

with $\mathrm{E}_{\mathrm{Na}}=74.25 \mathrm{mV}$, and $\bar{g}_{\mathrm{Na}}=110 \mathrm{nS} . m$ is the activation gating variable and $h$ is the inactivation gating variable, both governed by a first order equation:

$$
\frac{d x}{d t}=\frac{x_{\infty}(V)-x}{\tau_{x}(V)}
$$

Steady-state values and time constants of $m$ and $h$ are expressed as:

$$
\begin{aligned}
& m_{\infty}(V)=\frac{1}{\left(1+e^{-\frac{V+38.60}{4.12}}\right)} \\
& \tau_{m}(V)=1.6\left[\frac{1-\frac{0.2}{1.6}}{1+e^{\frac{V+29}{5.5}}}+\frac{0.2}{1.6}\right] \\
& h_{\infty}(V)=\frac{1-0.011}{\left(1+e^{\frac{V+48.99}{7.28}}\right)}+0.011 \\
& \tau_{h}(V)=17\left[\frac{1-\frac{2.8}{17}}{1+e^{\frac{V+31}{6.5}}}+\frac{2.8}{17}\right]
\end{aligned}
$$

Figure 1 shows the voltage-dependence of $m$ and $h$ steady-state values and time-constants. The parameters in equations (3) were determined to obtain a good fit of sodium current in spinal motor neurons. These measurements are in progress in the Laboratory of Biophysics (Department of Neuroscience, University of Rome "Tor Vergata", Italy). To identify the parameters of sodium current we used some preliminary results on spinal motor neurons of nontransgenic mice. Results of the identification of the voltage-clamp curves are shown in Figure 2. 

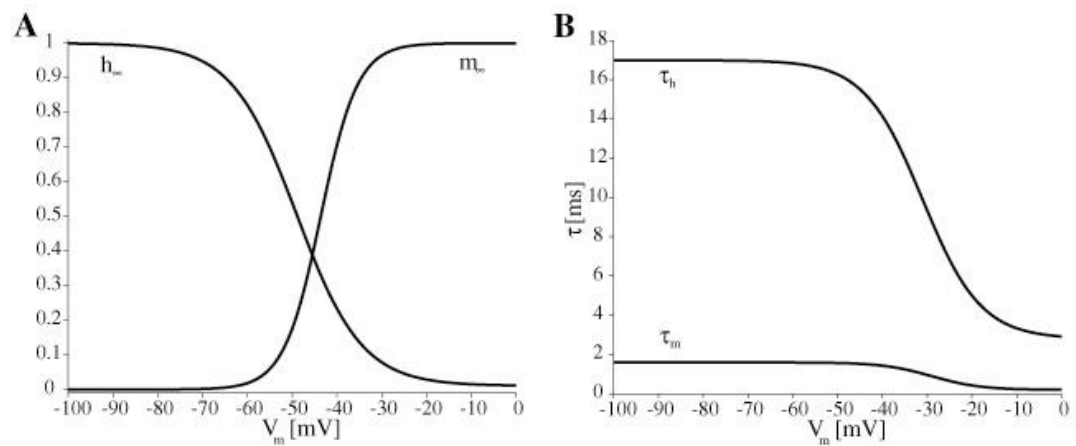

Figure 1: Steady-state values (A) and time constants (B) of fast sodium current activation and inactivation gating variables.
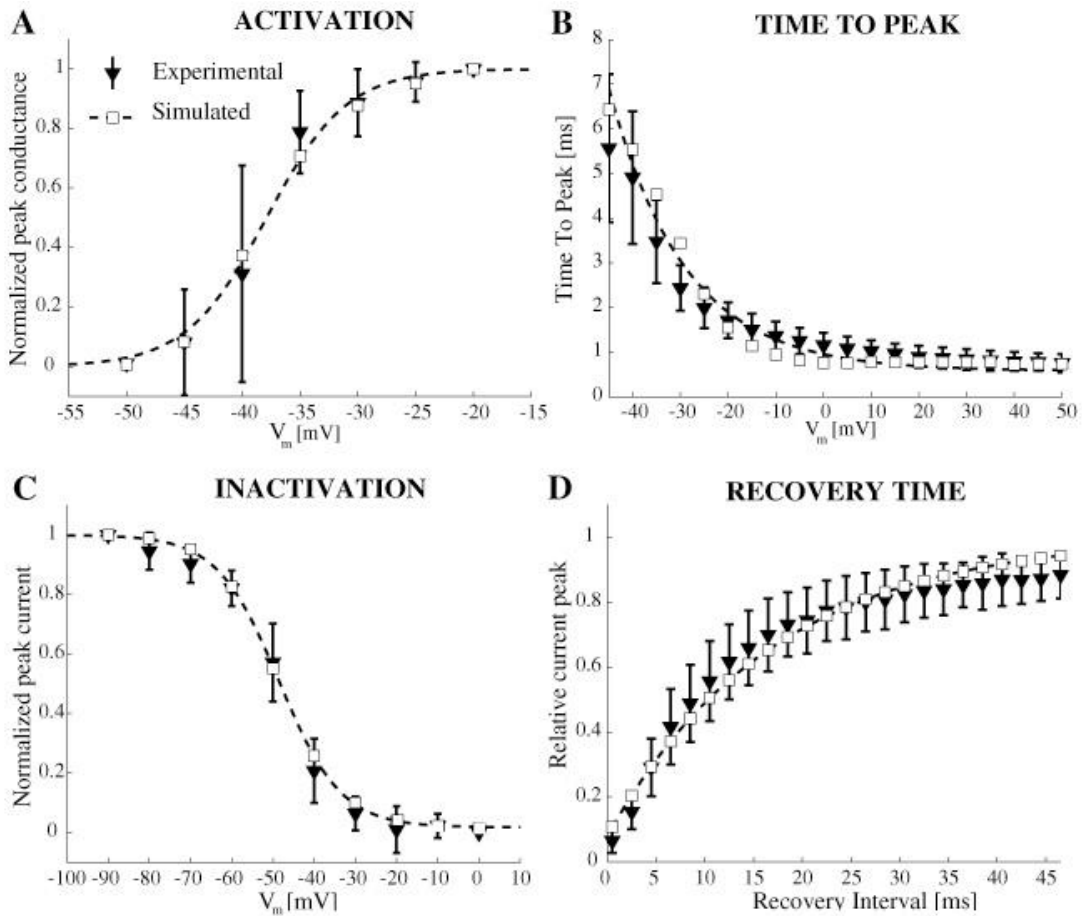

Figure 2: Fitting of experimental curves for fast sodium current: activation (A), time to peak (B), inactivation (C), and recovery from inactivation (D). 
After the identification of the parameters a $+5 \mathrm{mV}$ shift of the activation curve was necessary to fit experimental values of the threshold for action potential generation.

\subsection{Formulation of the mathematical model}

The model is based on a single-compartment Hodgkin-Huxley formulation [7] and it includes several ionic currents: the fast and the persistent sodium currents, the delayed-rectifier and the inactivating (A-type) potassium currents, two $\mathrm{Ca}^{2+}$ dependent potassium currents, the L-type calcium current, some background $\left(\mathrm{I}_{\mathrm{NaB}}, \mathrm{I}_{\mathrm{CaB}}\right)$ and some pump currents $\left(\mathrm{I}_{\mathrm{NaK}}, \mathrm{I}_{\mathrm{CaP}}\right.$, and $\left.\mathrm{I}_{\mathrm{NaCa}}\right)$. Along with these currents, the model contains a simplified description of an intracellular calmodulin calcium buffer.

The membrane potential can be calculated by means of the differential equation:

$$
\frac{d V}{d t}=\frac{1}{C_{m}}\left(-\sum I_{\text {ionic }}+I_{\text {stim }}\right)
$$

where $\mathrm{V}$ is the membrane potential, $\mathrm{C}_{\mathrm{m}}$ is the total membrane capacitance, $\mathrm{I}_{\text {ionic }}$ are the ionic currents through the membrane channels and $\mathrm{I}_{\text {stim }}$ is the stimulating current. The individual ionic currents were modeled using the Hodgkin-Huxley equation:

$$
I_{\text {ionic }}=\bar{g} y\left(V-E_{\text {ionic }}\right)
$$

where $\bar{g}$ is the maximum conductance for the considered current, $\mathrm{y}$ is the product of one or more gating variables (raised to integer powers), and $E_{\text {ionic }}$ is the reversal potential for the ions passing through the channel. The gating variables are assumed to be first-order processes governed by the equation:

$$
\frac{d x}{d t}=\frac{x_{\infty}(V)-x}{\tau_{x}(V)}
$$

where $x_{\infty}$ is the steady-state value of the gating variable $x$ and $\tau_{x}$ is its time constant. The modeling of calmodulin buffer is reported elsewhere [8].

The model is derived from a recently published work reproducing firing properties of phrenic motor neurons [9]. Compared to that paper, we changed the model for fast sodium current, as previously described, and we adjusted potassium channel conductance to compensate for the elimination of delay potassium current.

To reproduce firing properties of spinal motor neurons, we introduced other minimal changes to the parameters of calcium-dependent potassium current (small type): the time constant of activation was reduced to $4 \mathrm{~ms}$ and its maximal conductance was set to $0.07 \mu \mathrm{S}$. 


\subsection{Simulations}

The model equations were implemented using Simulink (The MathWorks, Inc., Natick, MA) and numerically solved by using an algorithm based on a modified Rosenbrock formula of order 2 with a maximum step size of $0.01 \mathrm{~ms}$, the tolerance for integration was $0.5 \times 10^{-6}$. Measurements on simulated spike trains were performed following the criteria defined for experimental data in [3] (AP properties measured on the first spike and firing frequency calculated as the inverse of the first inter-spike interval).

\section{Results}

The model is able to reproduce the firing response to a current injection, as shown in Figure 3.

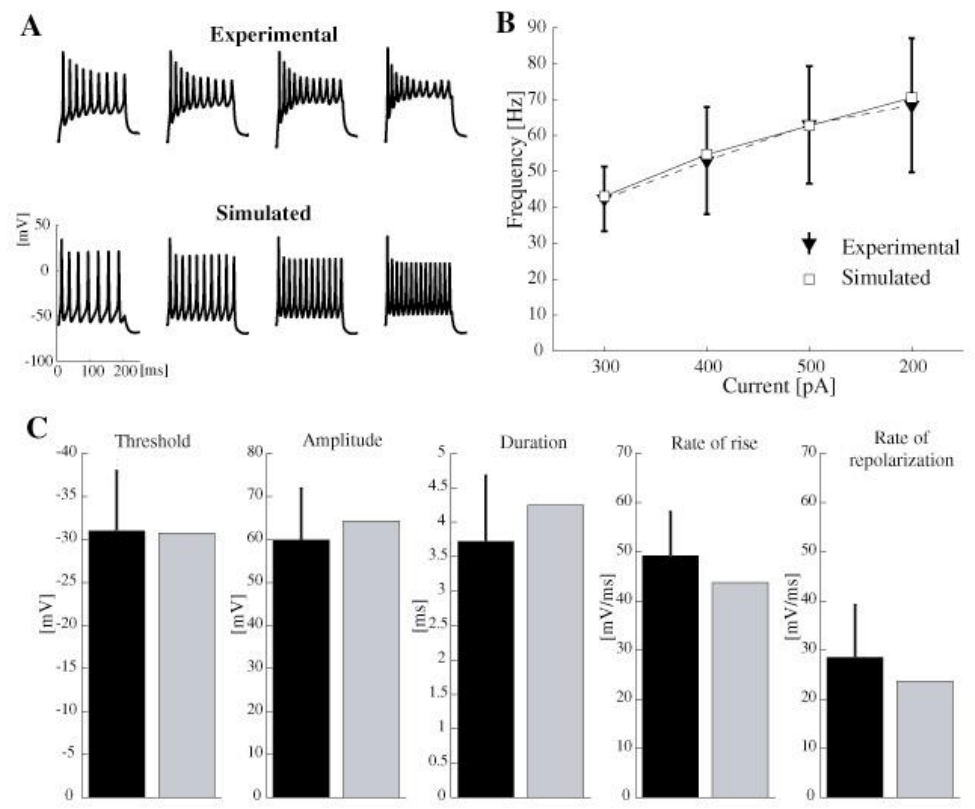

Figure 3: $\quad$ Firing properties in SOD1 motor neurons and in the model.

Figure $3 \mathrm{~A}$ shows representative membrane potential responses evoked by a current injection in SOD1 cells and in the model under current-clamp conditions. The relation between firing frequency and injected current in simulated spike trains fits the values measured in SOD1 motor neurons. Graph in Figure 3B shows the firing frequencies elicited with currents in the range of $+200 /+500 \mathrm{pA}$ in SOD1 cells (mean values and standard deviation) and in the model. Finally, 
there is a good correspondence between experimental and simulated AP properties (see Figure 3C).

\section{Discussion}

We have developed a numerical simulator for the mouse spinal motor neuron, suitable for the analysis of modifications at the ionic current level that can underlie neuron hyperexcitability in transgenic motor neurons.

Our model is largely based on a model of rat phrenic motor neuron recently proposed by Amini et al. [9]. The main modification concerned the fast sodium current. We decided to identify our model of this current in order to fit preliminary electrophysiological data from the Laboratory of Biophysics (Department of Neuroscience, University of Rome "Tor Vergata", Italy) for nontransgenic mouse spinal motor neurons because a) the fast sodium current has a fundamental role in action potential generation, hence in cell excitability, a correct formulation for this current is therefore crucial for studying motor neuron excitability b) in the Amini model, potassium and calcium currents were derived from phrenic motor neurons electrophysiological data, whereas fast sodium current description was adjusted from older experimental measures from bullfrog sympathetic ganglion cell [10], identification from the specific cell type of our interest was therefore needed. Since wild-type (non-transgenic) and transgenic SOD1 motor neurons showed similar firing frequency and AP characteristics [3], sodium current measures from wild-type cells seem suitable to also describe SOD1 behaviour. We did not take into account in our model the delay potassium current that was present in the Amini model; in fact we did not have any experimental evidence that this current exists in spinal motor neurons.

Our results indicate that our HH-type model, while semi-quantitative in its nature, captures the essential dynamic feature of spinal motor neuron excitability. It must be noted, however, that model validation was performed on a single set of experimental data. This should be considered a model limitation since its robustness remains to be evaluated.

However, the implemented model offers a powerful tool to investigate modifications in conductance and/or kinetics of the voltage-dependent currents which can explain the alterations observed in mutant motor neurons. Two analysis approaches can be profitably combined: a) a sensitivity analysis performed on the model by simulations will give clues to identify which current modifications could produce the observed effects on firing properties b) voltage clamp characterization of mutant currents will provide mathematical formulation for such currents to be inserted in the model to identify the consequence of pathology-induced current mutations at the action potential level.

\section{References}

[1] Rosen D.R., Siddique T., Patterson D., Figlewicz D.A., Sapp P., Hentati A., Donaldson D., Goto J., Regan J.P. \& Deng H.X., Mutations in Cu/Zn superoxide dismutase gene are associated with familial amyotrophic lateral sclerosis. Nature, 362, pp. 59-62, 1993. 
[2] Tu P.H., Raju P., Robinson K.A., Gurney M.E., Trojanowski J.Q. \& Lee V.M., Transgenic mice carrying a human mutant superoxide dismutase transgene develop neuronal cytoskeletal pathology resembling human amyotrophic lateral sclerosis lesions. PNAS, 93(7), pp. 3155-60, 1996.

[3] Pieri M., Albo F., Gaetti C., Spalloni A., Bengtson C.P., Longone P., Cavalcanti S. \& Zona C., Altered excitability of motor neurons in a transgenic mouse model of familial amyotrophic lateral sclerosis. Neuroscience Letters, 351(3), pp. 153-156, 2003.

[4] Zanette G., Tamburin S., Manganotti P., Refatti N., Forgione A. \& Rizzuto N., Different mechanisms contribute to motor cortex hyperexcitability in amyotrophic lateral sclerosis. Clinical Neurophysiology, 113(11), pp. 1688-1697, 2002.

[5] Gao B.X. \& Ziskind-Conhaim L., Development of ionic currents underlying changes in action potential waveforms in rat spinal motoneurons. Journal of Neurophysiology, 80, pp. 3047-3061, 1998.

[6] Hess D. \& El Manira A., Characterization of a high-voltage-activated IA current with a role in spike timing and locomotor pattern generation. PNAS, 98(9), pp. 5276-5281, 2001.

[7] Hodgkin A.L. \& Huxley A.F., A quantitative description of membrane current and its application to conduction and excitation in a nerve. Journal of Physiology, 117, pp. 500-544, 1952.

[8] Athanasiades A., Clark J.W., Ghorbel F. \& Bidani A., An ionic current model for medullary respiratory neurons. Journal of Computational Neuroscience, 9(3), pp. 237-257, 2000.

[9] Amini B., Bidani A. Zwischenberger J.B. \& Clark J.W., A model of the rat phrenic motor neuron. IEEE Transactions on Biomedical Engineering, 51(7), pp. 1103-1114, 2004.

[10] Schild J.H., Khushalani S., Clark J.W., Andresen M.C., Kunze D.L. \& Yang M., An ionic current model for neurons in the rat medial nucleus tractus solitarii receiving sensory afferent input. Journal of Physiology, 469, pp. 341-63, 1993. 